\title{
Effects of Chain Branching on Crystal Morphology and Structure of Polyethylene Single Crystal
}

\author{
Qiang Fu, Ya Peng, Qin Zhang, Eric T. Hsieh,* \\ and Chung C. Tso* \\ Department of Polymer Science \& Materials, Sichuan University, \\ Chengdu, Sichuan 610065, People's Republic of China \\ * Philips Petroleum Company, Bartlesville, Oklahoma 74004, U.S.A.
}

(Received April 30, 1999)

\begin{abstract}
We have successfully grown single crystal from low molecular weight metallocene short chain branched polyethylene (SCBPE) samples. The branching content and crystallization temperatures have a big effect on lateral habits. Lozenge, dendritic, $\{100\}$ face rounded and disk-like crystal morphologies have been found as branching content increase. The hexane side chain is included in the crystal lattice as suggested from the election diffraction and crystal thickness, for highly branched sample.

KEY WORDS Metallocene Short Chain Branched Polyethylene/ Single Crystal/ Morphology /
\end{abstract}

Much work has been done on solution crystallization of linear polyethylene (LPE) single crystals, ranging from the crystal structure, crystal morphology, growth kinetics and mechanism. ${ }^{1-10}$ The lamellar nature of polyethylene single crystals grown from dilute solution is well known. The well faceted lateral habits can be obtained from dilute solutions at relatively low crystallization temperature. The increasing curvature of nominally $\{100\}$ faces can be seen as the crystallization temperature increase or crystallization in poorer solvent, such as dodecane or heptyl acetate. The orthorhombic packing of LPE single crystals was found for all cases of solution crystallization. The nature of fold surface and the rounding of habits (thermally roughing or interrupted roughening) of PE single crystals are tow long-standing and much debated subjects in polymer science.

For the crystallization of copolymer, such as short chain branched polyethylene, the location of branching is another central issue, whether the branches are incorporated in the crystal lattice or they are in fact preferentially excluded from the crystal lattice as assumed in Flory's theory of copolymer crystallization. ${ }^{11}$ Irregularity in the lateral habits of branched $\mathrm{PE}$ single crystals has been reported by Kawai et al., ${ }^{12}$ Holdsworth and Keller, ${ }^{13}$ respectively. Marks and his coworkers employed model ethylene copolymers (hydrogenated polybutadienes, HPB) with very narrow molecular weight distribution and nearly random distributions of side branches to do the solution crystallization, ${ }^{14}$ and large expansions found for the basal plane of HPB crystals has led to the idea that the molecules undergo a crystal-crystal transformation to a hexagonal lattice. $^{15}$

This paper is: (1) to record some experimental data of solution crystallization from metallocene short chain branched polyethylene (SCBPE) with low molecular weight for the first time, and (2) conform further the location of side branches (included in or excluded from crystal lattice). The work is made possible based on the recent availability of metallocene catalyst and crossfractionation techniques for short chain branched PE. Compared with Ziegler-Natta copolymers, these me- tallocene SCBPEs set a new standard for polymer purity with narrow molecular weight and more uniform comonomer sequence distributions and make it possible to use low molecular weight fractions to grow nice single crystal from solution.

\section{EXPERIMENTAL}

Materials

Hexane-branched metallocene SCBPEs were provided by Phillips Petroleum Company. Molecular characteristics are listed in Table I. All the samples have been cross-fractionated before used. The molecular weight distribution for all the samples are quite narrow $\left(M_{w} / M_{n}<1.2\right)$. The branching content among molecules can be considered intermolecularly homogeneous, but intra-molecularly random distributed after being cross-fractionated. ${ }^{16}$

\section{Solvent}

p-Xylene was chosen as solvent for solution crystallization of SCBPE, as in the case of linear PE. The solvent was used before purification after being distilled.

\section{Sample Preparation}

Suspensions of polymer crystals in a solvent were prepared as follows. An appropriate weight SCBPE sample was first dissolved in the solvent by heating for two hours to $120^{\circ} \mathrm{C}$ in xylene to completely destroy all the crystal nuclei. Then crystallization for $1000 \mathrm{~min}$ in an oil bath was regulated to $+/-0.3^{\circ} \mathrm{C}$ at preset temperatures.

Table I. Molecular characteristics of samples

\begin{tabular}{ccccc}
\hline Fraction ID & $M_{n}$ & Mol\% & $\begin{array}{c}\text { Molecular } \\
\text { length } / \AA\end{array}$ & $M_{w}$ \\
\hline A55 & 1,830 & 1.89 & 225 & 2,150 \\
B71 & 2,720 & 1.67 & 345 & 3,270 \\
C81 & 4,130 & 0.92 & 510 & 4,730 \\
D91 & 5,160 & 0.39 & 630 & 5,910 \\
\hline
\end{tabular}

${ }^{\text {a }}$ Molecular length is calculated from molecular weight. 


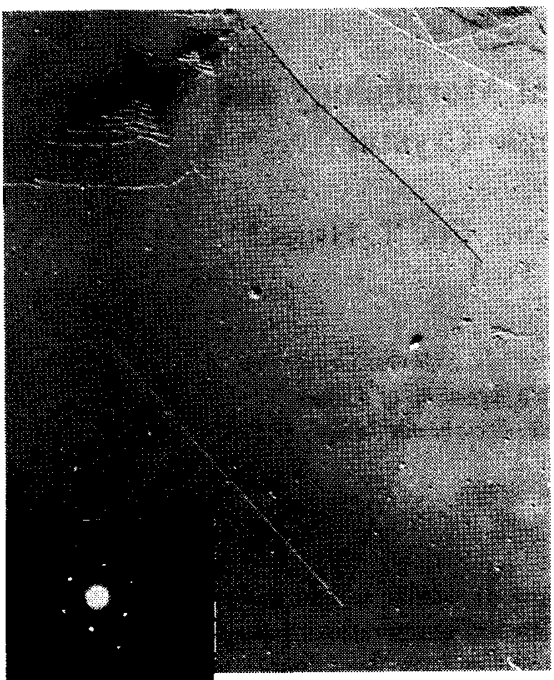

(a)

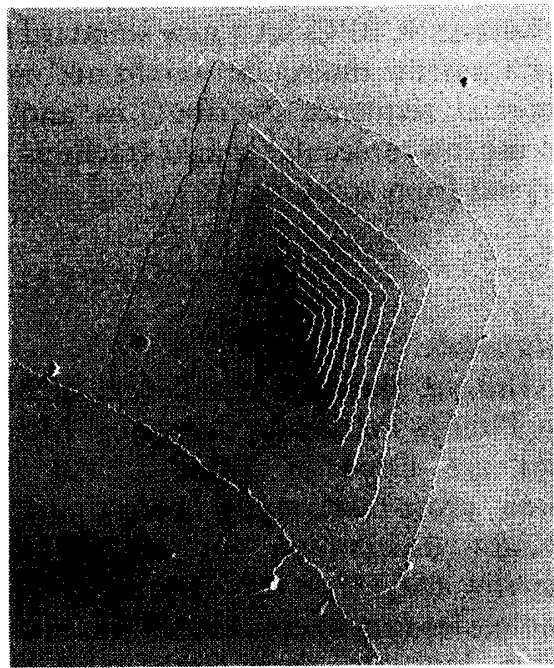

(c)

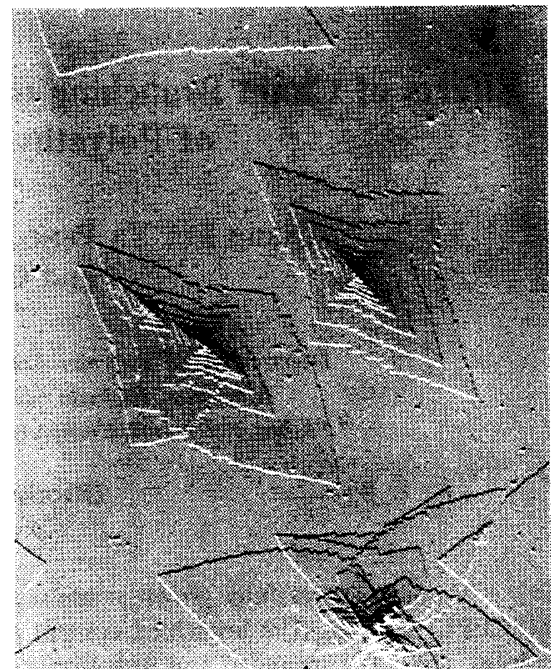

(b)

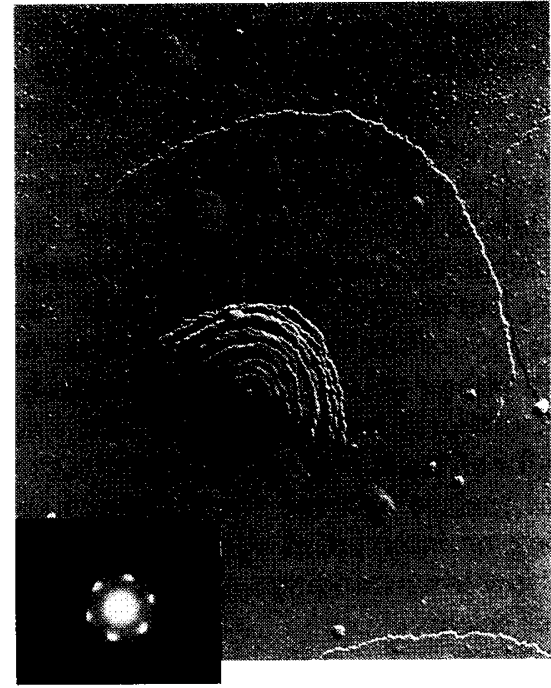

(d)

Figure 1. Single crystal of metallocene SCBPE grown from $0.2 \%$ solution in xylene at 80 C. Branching content: (a) $0.39 \%$; (b) $0.92 \%$; (c) $1.67 \%$; and (d) $1.89 \%$.

\section{Electron Microscopy and Diffraction}

Samples for electron microscopy and diffraction were deposited on carbon substrates before being shadowed and transferred to electron microscope grids. The prepared polymer crystal samples were examined via a JEOL JEM-1200EXII transmission electron microscope (TEM) operated at $120 \mathrm{kV}$ accelerating potential. To prevent the radiation damage as much as possible, very low illumination was used by keeping the beam spread and operating at low current.

\section{RESULTS AND DISCUSSION}

\section{Crystal Habits}

Figure 1 shows the single crystals of SCBPEs grown from $0.2 \%$ solution in the xylene at $80^{\circ} \mathrm{C}$. Compared with single crystals of branched PE reported previously, where the chief characteristics are highly irregular profiles and relatively small size, ${ }^{12-15}$ it is interesting to note following features of our SCBPE single crystals: (1) They exhibit the common lamellar growth habit; (2) As branching content increase from $0.39 \mathrm{~mol} \%$ to 1.89 mol\% (note the molecular weight decreases as branching content increases), morphologies changes from lozengen, dendritic, to $\{100\}$ faces rounded lozenge, and becomes disk-like profile and finely serrated character of the periphery, which is very similar to single crystal of polychlorotrifluoroethylene grown from a $0.1 \%$ solution in low molecular-weight polychlorotrifluoroethylene. ${ }^{17}$ And also the crystal size is big (around $2-3 \mathrm{um}$ ), probably because of low molecular weight and low branching content compared with branched PE used previously ${ }^{12-15}$; (3) For sample A55, which has the highest branching content, the crystal habit changed as crystallization temperature was investigated. As shown in Figure 2, the crystal shape does not change much when crystallization temperature is less than $83^{\circ} \mathrm{C}$. However, the crystal becomes highly elongated when crystalliza- 


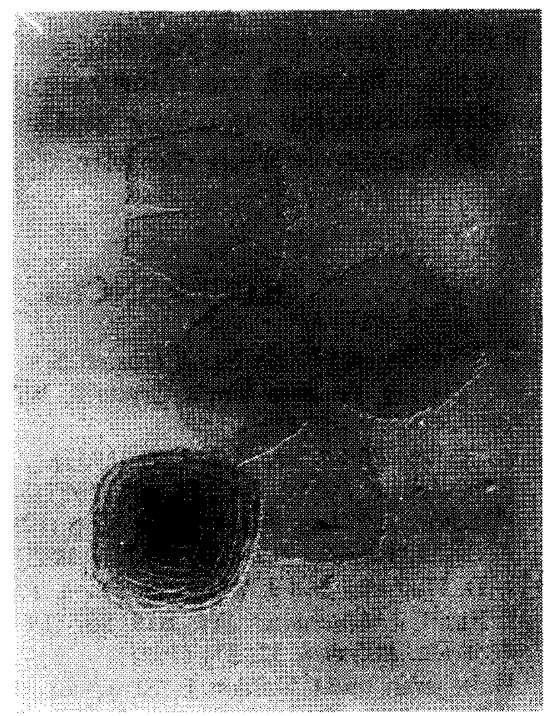

(a)

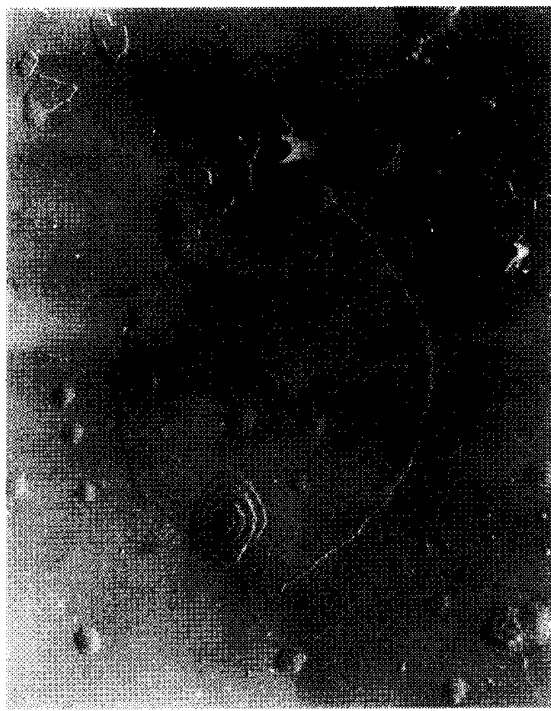

(c)

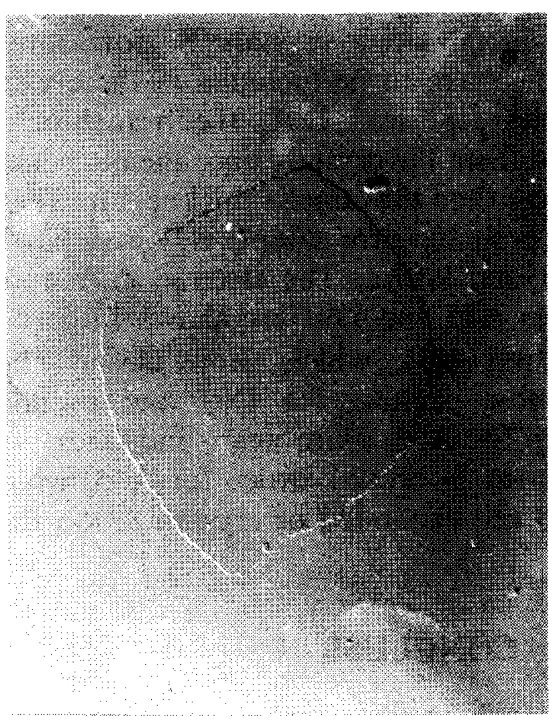

(b)

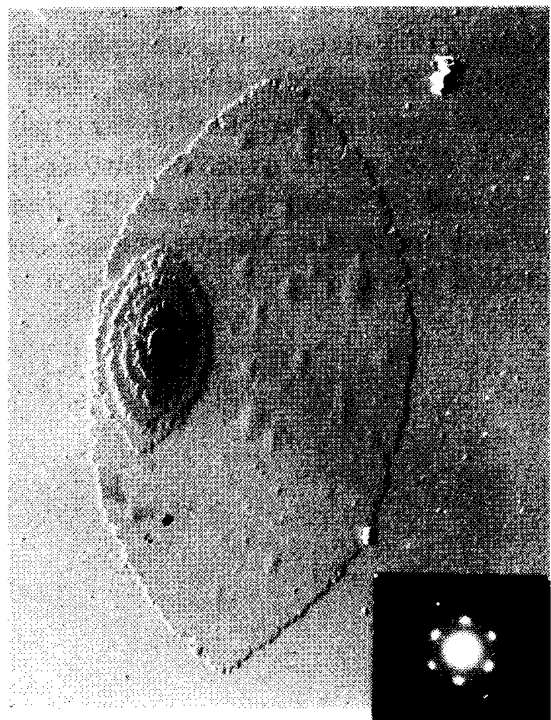

(d)

$500 \mathrm{~nm}$

Figure 2. Single crystal from sample A55 at different temperature. (a) $55^{\circ} \mathrm{C}$; (b) $65^{\circ} \mathrm{C}$; (c) $75^{\circ} \mathrm{C}$; (d) $83^{\circ} \mathrm{C}$.

tion temperature is above $83^{\circ} \mathrm{C}$. There is a sharp jump of axial ratio $a / b$ at crystallization temperature of $83^{\circ} \mathrm{C}$, as can be seen in Figure 3. There thus may be a growth mechanism change around $83^{\circ} \mathrm{C}$. No truncated habit was found for samples B71, C81, and D91 in the crystallization temperature range studied (goes up to $\left.84^{\circ} \mathrm{C}\right)$.

\section{Included and Excluded Model}

The side branching larger than octane excluded from out of crystal lattice, and branching less than ethyl is included in the crystal. It is much debated that branches between ethyl and octane are excluded or included in the crystal. We measured the thickness of single crystals to know the location of branching. At the same crystallization temperature $\left(80^{\circ} \mathrm{C}\right)$, though the crystal habits change as the branching increases, the lamella thickness does not change much (around 150-200 , calculated directly from the TEM pictures). Since the molecular

Polym. J., Vol. 32, No. 1, 2000

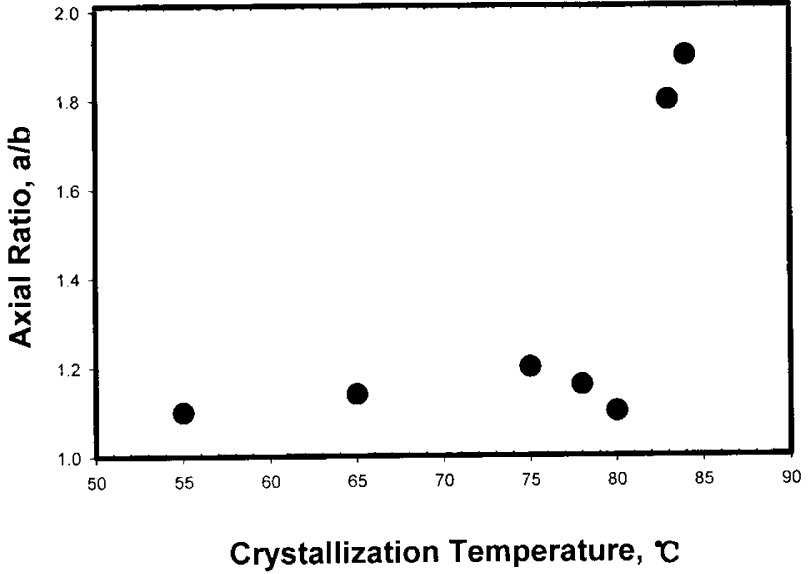

Figure 3. Variation of axial ratio, $a / b$, with crystallization temperature for sample A55. 
length is only $225 \AA$ for samples of A55, molecular chain must be extended in the single crystals. So for sample A55, some branches have to be included in the crystal lattice. For samples C81 and D91, the branching is most likely rejected from the crystals since the molecular length is much larger than the lamella thickness for these two samples. The branching for sample B71 may partly involved in the crystal lattice. The election diffraction patterns of samples A55 and D91 are shown in corner of Figures 1(a) and 1(d), which show clearly that typical orthorhombic packing for D91 and pseudohexagonal packing for A55. Here again indicates that side chain is included in crystal lattice for A55, which makes crystal unit cell expanded or crystallization in a new form.

\section{CONCLUSION}

The effects of branching on crystal habit and structure of polyethylene have been investigated. The primary observation is a general trend toward increasing irregularity as branching goes up. Lozenge, dendritic, $\{100\}$ face rounded and disk-like crystal morphologies have been found when branching increases, but is truncated. The hexane side chain is included in the crystal lattice for sample A55. It is worth to study further the nature of fold surface and rounding habits of PE single crystals using these low molecular weight metallocene SCBPEs with controlled branching.
Acknowledgments. The authors express great thanks to Prof. Stephen Cheng for the useful discussion and comments. This work was jointly supported by National Science Foundation of China and a Grant-in-Aid for Scientific Research from the Ministry of Education of China.

\section{REFERENCES}

1. S. J. Organ and A. Keller, J. Master. Sci., 20, 1571, 1586 (1985).

2. P. H. Geil, 'Polymer Single Crystals', Wilcy, New York, N.Y., 1963.

3. A. Keller, Philos. Mag., 2, 1171 (1957).

4. P. H. Till, J. Polym. Sci., 24, 301 (1957).

5. F. Khoury, Polym. Prepr., Jpn., 31, 5 (1982).

6. A. Toda, Faraday Discuss., 95, 129 (1993).

7. J. D. Hoffman and J. Weeks, J. Chem. Phys., 42, 4301 (1965).

8. J. Rault, J. Macromol. Sci., B15, 567 (1978).

9. A. Toda, Polymer, 32, 771 (1991)

10. H. Beijeren, and I. Nolden, I. "Structure and Dynamics of surface II," Topics in Current Physics, Springer, Berlin, 1987, Chapter $7, \mathrm{p} 43$.

11. P. Flory, J. Trans. Faraday Soc., 51, 848 (1955).

12. T. Kawai, K. Ujihara, and H. Maeda, Markromol. Chem., 132, 87 (1970).

13. P. J. Holdsworth and A. Keller, J. Polym. Sci., Polym. Lett. Ed., 5, 605 (1967).

14. B. S. Marks and S. H. Carr, J. Appl. Phys., 53, 6526 (1982).

15. B. S. Marks and S. H. Carr, J. Polym. Sci., Polym. Phys. Ed., 23, 1563 (1985).

16. E. Hsieh, C. Tso, Q. Fu, and S. Z. D. Cheng, J. Macromol. Sci.-Phys., B36, 615 (1997).

17. J. D. Barnes and F. Khoury, J. Res. Nat. Bur. Std. (U.S.), 78A, 363 (1974) 